\title{
Focusing properties of an axicon pair
}

\author{
Marc Couture \\ Module Science et Technologie, Télé-universite, Québec, Canada \\ Michel Piché \\ Centre de recherche en optique et laser (COPL), \\ Département de physique, Université Laval, Québec, Canada
}

Note. This is the final, revised draft of the paper published in Canadian Journal of Physics, 1993, 71(1-2), 70-78. doi:10.1139/p93-011

\begin{abstract}
The focusing properties of a so-called reflaxicon (a combination of a diverging and a converging axicon) are studied both theoretically and experimentally. Calculations of intensity distributions produced by this system are made by evaluating the Kirchhoff-Fresnel diffraction integral, first. by means of an approximate technique, the stationary phase method, then by a more exact numerical method. The calculations are presented for various planes along the axis of the axicons. The effects of the presence of the supporting mount of the axicons and of some important misalignments of the system on the distributions is also investigated. Experimental results of actual intensity distributions produced by focusing a near-fundamental Gaussian beam by such a system are also presented and are seen to be in fair agreement with numerical calculations. Such calculations would be valuable in many applications for predicting important characteristics (e.g., peak intensity, length of the focal line, degree of asymmetry) of the intensity distributions formed by optical systems containing an axicon pair as the focusing component.
\end{abstract}

\section{Résumé}

Les propriétés de focalisation d'un système optique formé d'un axicon convergent suivi d'un axicon divergent font l'objet d'une étude théorique et expérimentale. Les distributions d'intensité théoriques produites par ce système sont déterminées en résolvant l'intégrale de diffraction de Kirchhoff-Fresnel, d'abord de façon approximative, par la méthode de la phase stationnaire, puis, d'une manière plus exacte, par le recours au calcul numérique. Ces distributions sont illustrées pour divers plans situés le long de l'axe des axicons; on examine également l'effet de la présence de la monture des axicons et des principale erreurs d'alignement du système. On présente ensuite le résultat de mesures de distributions d'intensité produites en focalisant un faisceau gaussien quasi-fondamental à l'aide d'un système semblable; on constate un bon accord avec les résultats des calculs numériques. De tels calculs présentent un intérêt pour nombre d'applications : ils permettraient de prédire certaines caractéristiques importantes (par exemple, l'intensité maximale, la longueur de la ligne focale, le degré d'asymétrie) des distributions d'intensité produites par des systèmes optiques utilisant une paire d'axicons comme éléments focalisants 


\section{Introduction}

The term "axicon," which means literally "axial image" was introduced by McLeod [1] to describe any axially symmetric optical component for which the image of a point source located on the axis extends on a line along the same axis. In that sense, an axicon can be considered as a lens with over-corrected spherical aberration. Of all the possible shapes that can produce this type of focusing, the cone is the one that has attracted most attention, so that nowadays the word axicon generally stands for a conical optical component. One finds refracting and reflecting axicons, as well as converging and diverging ones, the latter forming a virtual line image of a point source.

Optical systems containing a single axicon have been proposed for applications in various areas, to name a few: spectroscopy [2], stable-unstable resonator mode couplings [3], interferometry, collimation of atomic beams [6], optical-fiber treatment [7], ophthalmology [8], and radial optical pumping $[9,10]$. In particular, the use of an axicon for the production of long plasma lines [11-14] appears promising.

A system formed by two reflecting axicons (a diverging axicon followed by a converging one) was designed by Edmonds [15], who called it "reflaxicon". This system also found some applications, notably in machining [16], unstable resonators [17], ophthalmology [18], and axial optical pumping ${ }^{1}$. Meanwhile, a few analyses of the focusing properties of axicons appeared in the literature, most of them concerned with a single axicon. Analytical expressions of axial and transverse intensity distributions were obtained for the cases of a point-source [19], a plane wave, and a Gaussian beam [20-24]. The basic focusing properties of the reflaxicon were also studied by means of elementary geometrical optics [15] and a ray-tracing program [25]; the latter case included an analysis of the effects of misalignments (tilt and decenter, i.e., relative lateral displacement of the axicons) on the shape of the wave fronts. It is to be noted that only two experimental results related to axicon focusing, both of very limited scope, were reported; a crude measure of the intensity profile of the ring produced by a combination of a reflaxicon and a lens [26], and some photographic plates obtained with a single axicon [24].

To obtain valuable knowledge of the focusing properties of the reflaxicon, which could be used to estimate some relevant characteristics of the intensity distributions (like, for instance, the maximum intensity or the width of the peaks) in a given optical system, it seemed important to pursue a detailed theoretical (numerical) analysis, based on physical optics, as well as a detailed experimental analysis of the intensity distributions produced by this still largely unknown optical component.

The system we chose to study, a pair of reflecting axicons, was designed for use at $10 \mu \mathrm{m}$ in an optical pumping configuration described elsewhere ${ }^{1}$. The same study could have been made with coated refractive components, but reflective (metallic) optical components have many advantages; they can be used at any mid- or far-IR wavelength; they are less prone and; contrary to the often-used (at $10 \mu \mathrm{m}$ ) Ge components, they can be aligned using a He-Ne laser beam. Another advantage is related to the particular shape of the (conical) axicons: they are fabricated by diamond-tip cutting machines that produce a surface already smooth to $\lambda / 40$ at $10 \mu \mathrm{m}$. Components made of other materials (or metallic ones for use at shorter wavelengths) require further polishing, which is extremely difficult for conical shapes.

\footnotetext{
${ }^{1}$. M. Couture and M. Piché manuscript in preparation.
} 


\section{Focusing of a Gaussian beam by an axicon pair with tilt and decenter: Geometrical optics and Kirchoff-Fresnel diffraction}

The axicon pair studied in this work is shown in Figure 1. It consists of a first $5 \mathrm{~cm}$ diameter diverging axicon (apex half-angle: $41^{\circ}$ ), and of a second converging axicon with a slightly lower half-angle $\left(40^{\circ}\right)$. This combination results in a $2^{\circ}$ focusing angle in the case of a plane wave incident on the axicons. For such a small focusing angle $2 \alpha$, where a is the difference in the angles of the two axicons, a beam of radius wand radius of curvature $r_{\mathrm{c}}$ results in a geometrical focal region of length $L$ given approximately by

$$
L \cong \frac{w\left[1+\left(a / 2 \alpha r_{c}\right)\right]}{2 \alpha-w / r_{c}}
$$

where $a$ is the inner radius of the annular beam in the exit plane of the axicons. For large values of $r_{\mathrm{c}}$ (corresponding to a collimated beam), $L$ reduces simply to $w / 2 \alpha$.
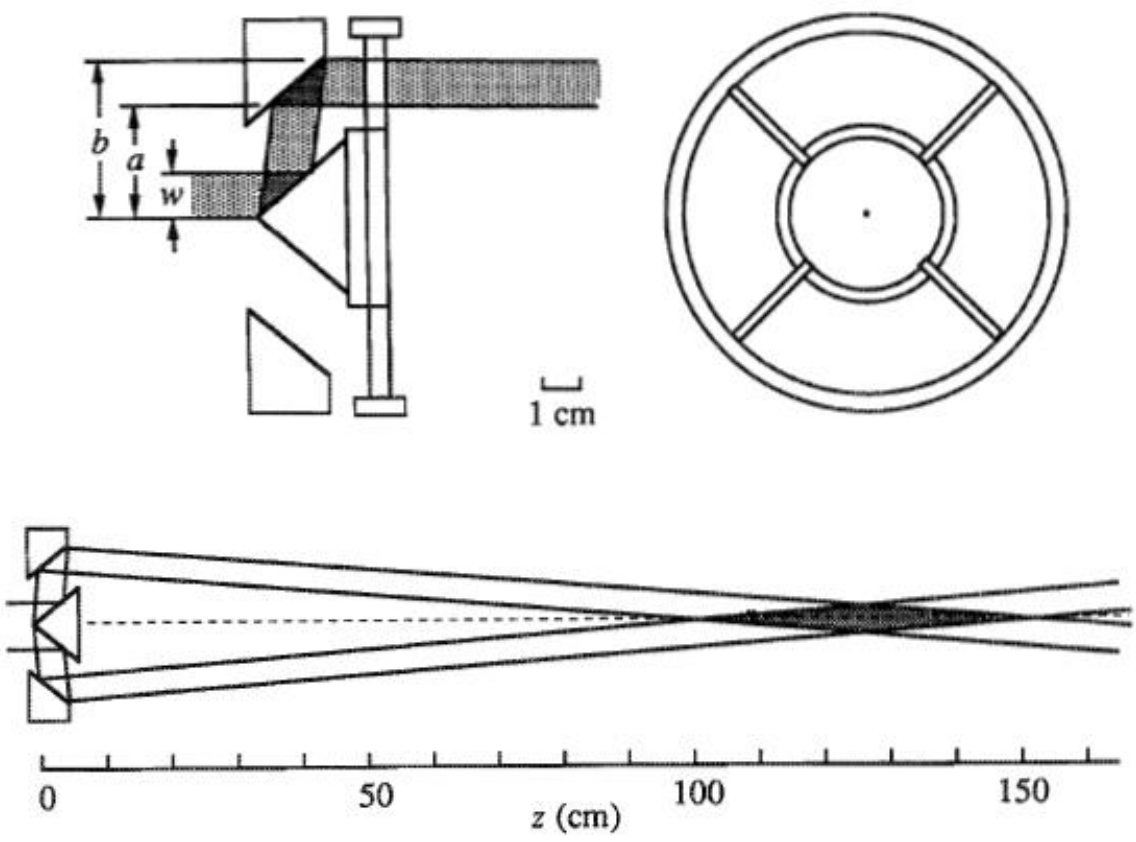

Figure 1. Lateral view of axicon pair, illustrating the notation (top left); front view of the diverging axicon and its mount (top right); location of the focal region (bottom).

We now consider three types of alignment errors, all three of great practical importance:

(i) decenter (relative lateral displacement of the two axicons),

(ii) tilt (relative angular displacement), and, in the case of an axially symmetric beam (1ike, for instance, a fundamental Gaussian beam),

(iii) lateral displacement of the beam center relative to the axis of the first axicon.

For the sake of simplicity and in view of the experimental results presented later, we restrict our study to the case where decenter and displacement lie in the same axis. To first order, one can neglect the effect of a misalignment along a given axis on the intensity distribution along an axis 
perpendicular to the first axis. Small misalignments along each axis can thus be studied independently.

The first step in the analysis is the transformation of the incident beam as it goes through the axicon pair; this is obtained by means of geometrical optics, which amounts to neglect of the small amount of diffraction occurring upon propagation over a few centimetres. If the beam incident on the first axicon is described by

$$
\Phi_{\mathrm{i}}(r, \theta)=A_{\mathrm{i}}(r, \theta) \exp \left[i f_{\mathrm{i}}(r, \theta)\right]
$$

one obtains simply, for the field at coordinates $\left(r_{0}, \theta_{0}\right)$ in the exit plane of the axicons,

$$
\Phi\left(r_{0}, \theta_{0}\right)=\left\{\begin{array}{l}
A_{i}\left(r_{0}-a, \theta_{0}\right)\left[\frac{r_{0}-a}{r_{0}}\right]^{1 / 2} \exp \left[i\left(f_{i}\left(r_{0}-a, \theta_{0}\right)-2 k \alpha r_{0}\right)\right], \quad a \leq r_{0} \leq b, \\
0, r_{0}<a \text { or } r_{0}>b
\end{array}\right.
$$

where $b$ is the outer radius of the second axicon.

By considering the change in the optical path, one can easily show that a small decenter $\Delta$ $(\Delta \ll b)$ along the $x$ axis adds a phase shift $\Delta f_{\mathrm{d}}$ given by

$$
\Delta f_{d} \cong k \Delta\left(\sin 2 \alpha_{i}+\sin 2 \alpha\right) \cos \theta_{0}
$$

where $k$ is the wave number and $\alpha_{i}$ the angle of the first (diverging) axicon. For $\alpha_{i}$ close to $45^{\circ}$ and small $\alpha$, the factor in parentheses is nearly equal to unity, so that one can write more simply in that particular case

$$
\Delta f_{d} \cong k \Delta \cos \theta_{0}
$$

A displacement $d$, again along the $x$ axis, of the center of the incident beam relative to the axis of the first axicon results in the replacement of the factor $r_{0}-a$ in the expressions for the amplitude $A_{\mathrm{i}}$ and the phase $f_{\mathrm{i}}$ in (3) by a new quantity $r^{\prime}$ given by

$$
r^{\prime}=\left[\left(r_{0}-a\right)^{2}+d^{2}-2 d\left(r_{0}-a\right) \cos \theta_{0}\right]^{1 / 2}
$$

Finally, one can show that, at least in the frame of the usual approximations involved in the formulation of the Kirchoff-Fresnel diffraction theory, a tilt of the axicons is mathematically equivalent to a combination of a decenter and a rotation of the optical axis past the axicons, so then it needs not to be treated separately.

Now, the field at a point $(r, \theta)$ in a given plane along the $z$ axis, past the axicons, is given, up to a constant phase factor, by the Kirchhoff-Fresnel integral

$$
A(r, \theta)=\frac{k}{z} \int_{a}^{b} \int_{0}^{2 \pi} \Phi\left(r_{0}, \theta_{0}\right) \exp \left[-i k S\left(r, \theta ; r_{0}, \theta_{0}\right)\right] r_{0} \mathrm{~d} r_{0} \mathrm{~d} \theta_{0}
$$

where $S\left(r, \theta ; r_{0}, \theta_{0}\right)$, with the usual (Fresnel) approximation, is given by

$$
S\left(r, \theta ; r_{0}, \theta_{0}\right) \approx z+\frac{r^{2}+r_{0}^{2}-2 r r_{0} \cos \left(\theta-\theta_{0}\right)}{2 z}
$$


Substituting (3), (4b), (5), and (7) into (6) and taking advantage of the integral representation of the Bessel functions, one can readily write the expression of the field at the observation point $(r, \theta)$ or $(x, y)$ in a plane located at a distance $z$ from the exit plane of the axicons, in the case of a Gaussian beam (radius of curvature $r_{\mathrm{c}}$ and beam radius $w$ ) incident on the axicons; the expression is

$$
\begin{array}{r}
A(r, \theta)=C_{0} \frac{k}{z} \exp \left[i k\left(z+\frac{r^{2}}{2 z}\right)\right] \int_{a}^{b} \exp \left\{\frac{\left(r_{0}-a\right)^{2}+d^{2}}{w^{2}}+i k\left[\frac{\left(r_{0}-a\right)^{2}+d^{2}}{2 r_{c}}+\frac{r_{0}^{2}}{2 z}-2 \alpha r_{0}\right]\right\} \\
\times\left[r_{0}\left(r_{0}-a\right)\right]^{1 / 2} J_{0}\left[k \Gamma\left(r_{0}\right)\right] \mathrm{d} r_{0}
\end{array}
$$

where $J_{0}\left[k \Gamma\left(r_{0}\right)\right]$ is a zero-order Bessel function whose complex argument is given by

$$
\Gamma^{2}\left(r_{0}\right)=\left[\frac{r_{0} x}{z}+\frac{d\left(r_{0}-a\right)}{r_{c}}-\Delta+i \frac{2 d\left(r_{0}-a\right)}{k w^{2}}\right]^{2}+\left[\frac{r_{0} y}{z}\right]^{2}
$$

The integral of (8) possesses no manageable analytical solution; one has to look for approximate analytical solutions or numerical integration techniques.

\section{Intensity distributions along the axis: Approximate solutions and numerical calculations}

The first method to be considered is the well-known stationary-phase method [27, 28].

According to its more general formulation [28], the result of an integration containing a rapidly oscillating factor is directly related to the value of the other factors of the integral and their derivatives evaluated at so-called critical points. Two such sets of points exist: the points where the first derivative of the phase of the oscillating factor vanishes, called points of stationary phase, and the integration limits. More precisely, it can be shown that an integral of the form

$$
\int_{a}^{b} g(r) e^{i k h(r)} \mathrm{d} r
$$

with $k \Delta h \gg$ 1, where $\Delta h$ is the range of the values of $h(r)$ in the interval $[a, b]$, may be expressed as a sum of powers of terms of the order of $(1 / k \Delta h)$, the leading terms of the series being

$$
\left[\frac{\pi}{2 k h^{\prime \prime}\left(r_{s}\right)}\right]^{1 / 2} g\left(r_{s}\right) e^{i\left[k h\left(r_{s}\right)-(\pi / 4)\right]}
$$

where $r_{\mathrm{s}}$ is a point where the phase $h(r)$ is stationary.

In the present case, described by (8), the oscillating part of the Bessel function combines with the argument of the exponential, resulting in the presence of two such points of stationary phase. If one considers only observation points located in the plane $\theta=0$ (or, equivalently, $y=0$ ), the locations of the stationary points are given by 


$$
r_{s}^{ \pm}=\frac{\alpha r_{c}+a \pm d}{1+\frac{r_{c}}{z}} \mp \frac{x}{1+\frac{z}{r_{c}}}
$$

One can easily show that these two points correspond physically to the origins of the rays lying in the same plane $\theta=0$ and intersecting at $x$ in the observation plane.

The next terms of the series expansion involve $h^{\prime}(a)$ and $h^{\prime}(b)$, the derivatives of the phase at the endpoints of integration, as well as the amplitudes $g(a)$ and $g(b)$ or, if the amplitude vanishes at an endpoint, its first derivative. These terms become dominant when, for a given value of the observation point $x$, no point of stationary phase lies within the limits of integration, which means that the observation point falls into the geometrical shadow region. The contribution of the upper limit of integration $b$ can be omitted if the width of the incoming Gaussian beam is small enough, namely, if $w$ is sufficiently lower than $(b-a)$, so that the amplitude of the field and its derivative are negligible at $r_{0}=b$, which is just the case for the particular system under study. Unfortunately, this is not true for the lower limit $a$, as the first derivative of the field distribution at the exit plane of the axicons diverges at $r_{0}=a$. While this divergence is not detrimental to the meaning of the integral, as it is easy to show that it possesses a well-defined finite value, it clearly reduces the interest of the method, unless one can appraise the effect of neglecting this contribution. First of all, one has to realize that this divergence is an artifact of the use of geometrical optics in the first stage of the calculation (propagation between the axicons). The actual distribution (which could be obtained in principle by the use of a full-fledged diffraction calculation taking into account the radius of the tip of the first axicon and the large angles involved) would not present any divergence at all; the field amplitude should grow from a very small value at $r_{0}=0$, which would become the lower limit of integration and where, due to circular symmetry, the first derivative of the field amplitude vanishes, to a value close (with small variations due to diffraction) to that predicted by geometrical optics in the region $r_{0}>a$. Thus, one can see the divergence in the derivative of the integrand as a result of the concentration of all the diffracted intensity, which should in fact be distributed over the region $r_{0}<a$, in the vicinity of this value of the radius. The omission of the contribution of the point $r_{0}=a$ in the stationary-phase method is in a sense equivalent to neglecting the effects of diffraction due to the tip of the first axicon and to the propagation between them. Considering the minute fraction of the incoming beam intensity hitting the tip of the axicon and the modest amplitude of the expected undulations occurring in a relatively short propagation distance (a few centimetres), this approximation should not affect the validity of the method further.

To estimate more precisely what the influence is of the intensity in the vicinity of $r=a$ on the intensity in the observation plane, we calculated how the peak intensity changes when one discards the corresponding points in a numerical integration (described below). We found that this contribution is indeed significant outside the focal zone; for instance, at $z=70 \mathrm{~cm}$, the contribution of the first $170 \mu \mathrm{m}$ wide annulus is comparable with that of the entire beam; the same is observed in the planes located past the focal zone. This is easy to understand if one considers the principle of the method; when there is no stationary point, the fast oscillations of the integrand cancel out, except at the endpoints, where the asymmetry in the first oscillation, which is easily seen to be proportional to the slope of $g(r)$ near $r=a$, can add an important contribution to the integral. However, the same calculation reveals that the contribution of the 
same area amounts to only a few percent in the focal zone, the dominant contribution coming from the points of stationary phase.

The result of the integration may thus be put equal to zero, at any point $x$ along the axis where decenter and lateral displacement may occur, for the term given by (11), or to the sum of two such terms according to the number of stationary points $(0,1$, or 2$)$ lying in the interval $[a, b]$. The overall accuracy of the method lies somewhere between those of geometrical optics and diffraction theory and, with the approximation discussed above, it should give meaningful results except in the near-axis parts of the distributions outside the focal zone. This is exactly what can be seen in Figure 2; the expected central peak (namely, the Arago spot) of the calculated distributions before and after the geometrical focal region does not show, while possessing a finite value inside that region.

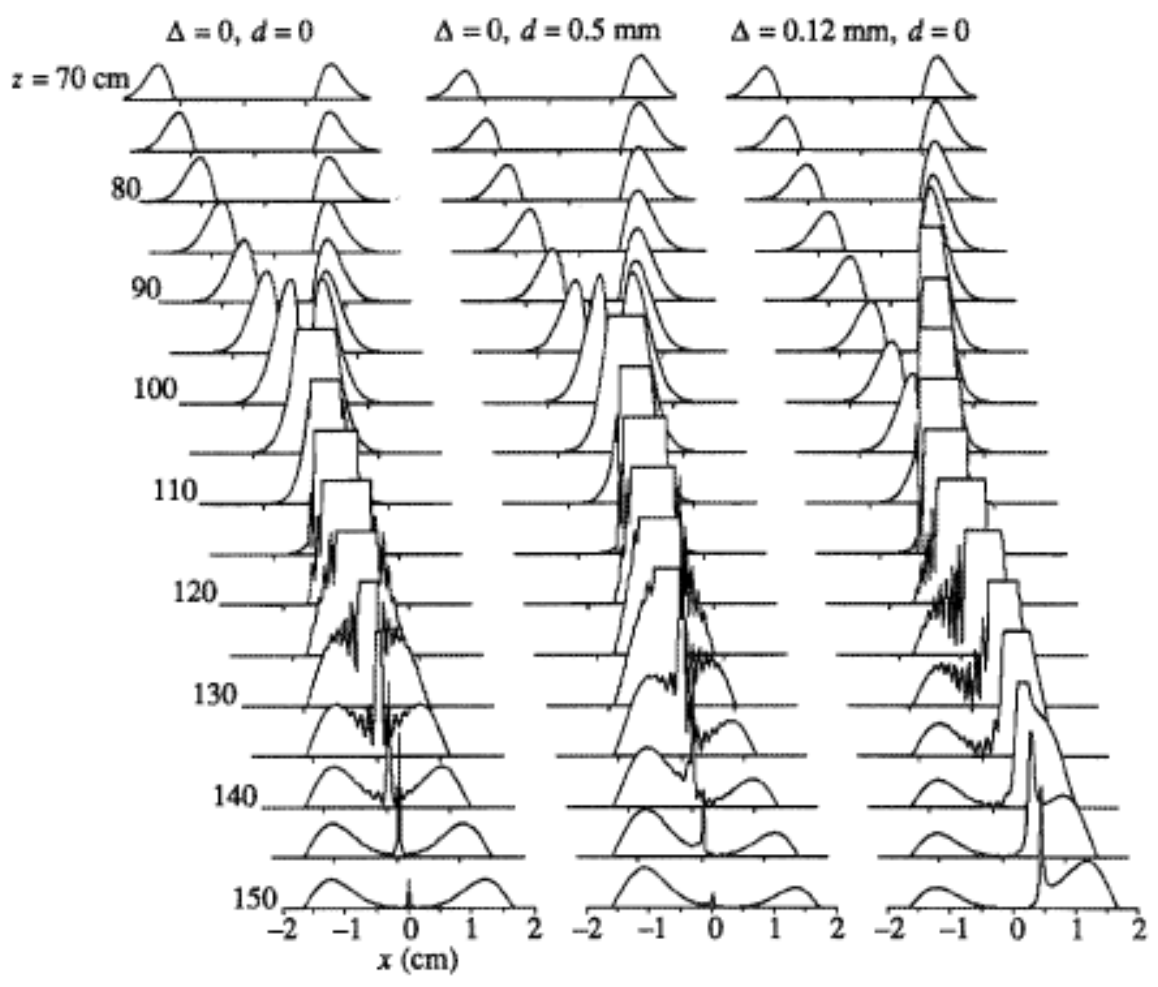

Figure 2. Global view of calculated intensity distributions along $\mathrm{x}$ axis for various planes, without decenter nor displacement (left), with $0.5 \mathrm{~mm}$ displacement (center) and with $0.12 \mathrm{~mm}$ decenter (right). Approximate analytical calculation by the phase stationary method.

In spite of its limitations, this method has the advantage of giving, with a minimum of calculation, a good description of some other features of the distributions, as will be revealed in a comparison with more exact (numerical) results. It can also be a useful tool for explaining or predicting the behaviour of the distributions as some parameters are changed, as well as a heuristic way to find a coarse value of these parameters when fitting numerical data to experimental results. For instance, one can use the results obtained by this method to estimate the actual amount of decenter and displacement from experimental measures of distribution. In particular, the fact that the asymmetry at the sides of the ring is inverted and the position of the peak intensity remains unchanged as one goes through the focal region in the case of 
displacement, contrary to what is observed in the case of decenter, gives good clues about the relative contributions of these two misalignments.

For a more accurate treatment one has to resort to numerical integration techniques. Two such methods were used at various stages of this work. The first is Gauss-Legendre integration, where care was taken to include a sufficient number of points for each oscillating cycle of the integrand. The second one is the so-called projection method [29], which consists in solving the Cartesian coordinates version of (8) by applying successively a numerical integration (again Gauss-Legendre) over one coordinate and a fast Fourier transform over the other. The advantage of this last method is that nonaxially symmetric elements, like the presence of the supporting mount of the first axicon or beams with astigmatism (different radii of curvature along perpendicular axes) can be included in the analysis without increasing calculation time. Furthermore, the observed similarity in the results from two essentially independent methods (applied to the symmetric case) is a good indication of their accuracy.

Figure 3 shows the results of the numerical calculations (Gauss-Legendre) of the same distributions as shown in Figure 2. Diffraction effects are now clearly visible; the central peak is present well before and after the geometrical focal region, and the profile of the ring shows undulations typical of propagation of $10 \mu \mathrm{m}$ truncated beams over a few tens of centimetres. The validity of the stationary phase method in the focal region can also be appreciated; it gives a good idea of the main qualitative features of the distributions, particularly the effect of the decenter and displacement, as well as a fair approximation of the intensity of the central peak, which may differ by, at most, a factor of 2 from the result of the numerical integration.

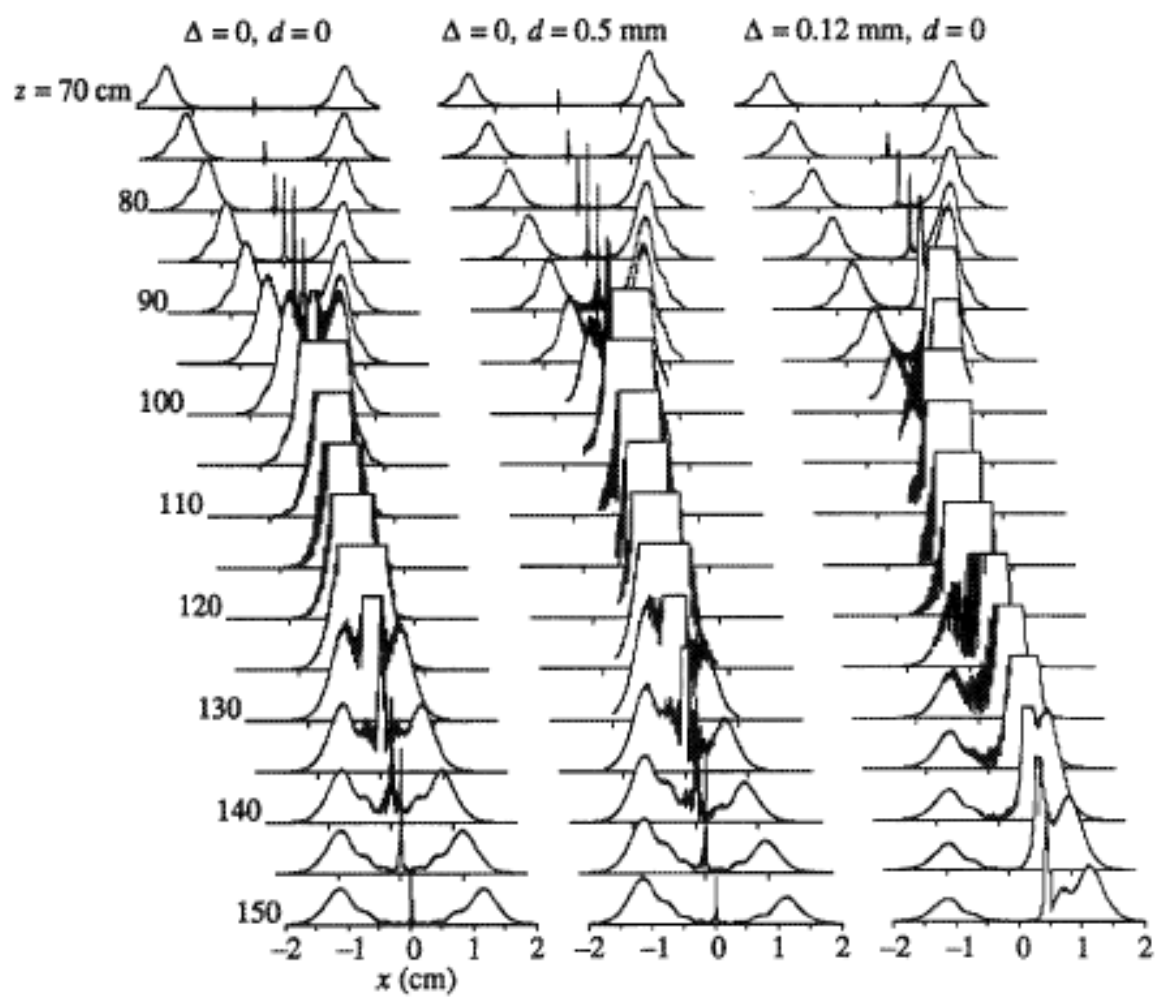

Figure 3. Same global view as in Figure 2, with same parameters, this time calculated by Gauss-Legendre numerical integration, neglecting the presence of the supporting mount of the first axicon. 
Figure 4, which gives two-dimensional views of the distributions in a particular plane $(z=90 \mathrm{~cm})$, illustrates the effect of increasing decenter and lateral displacement of the beam.
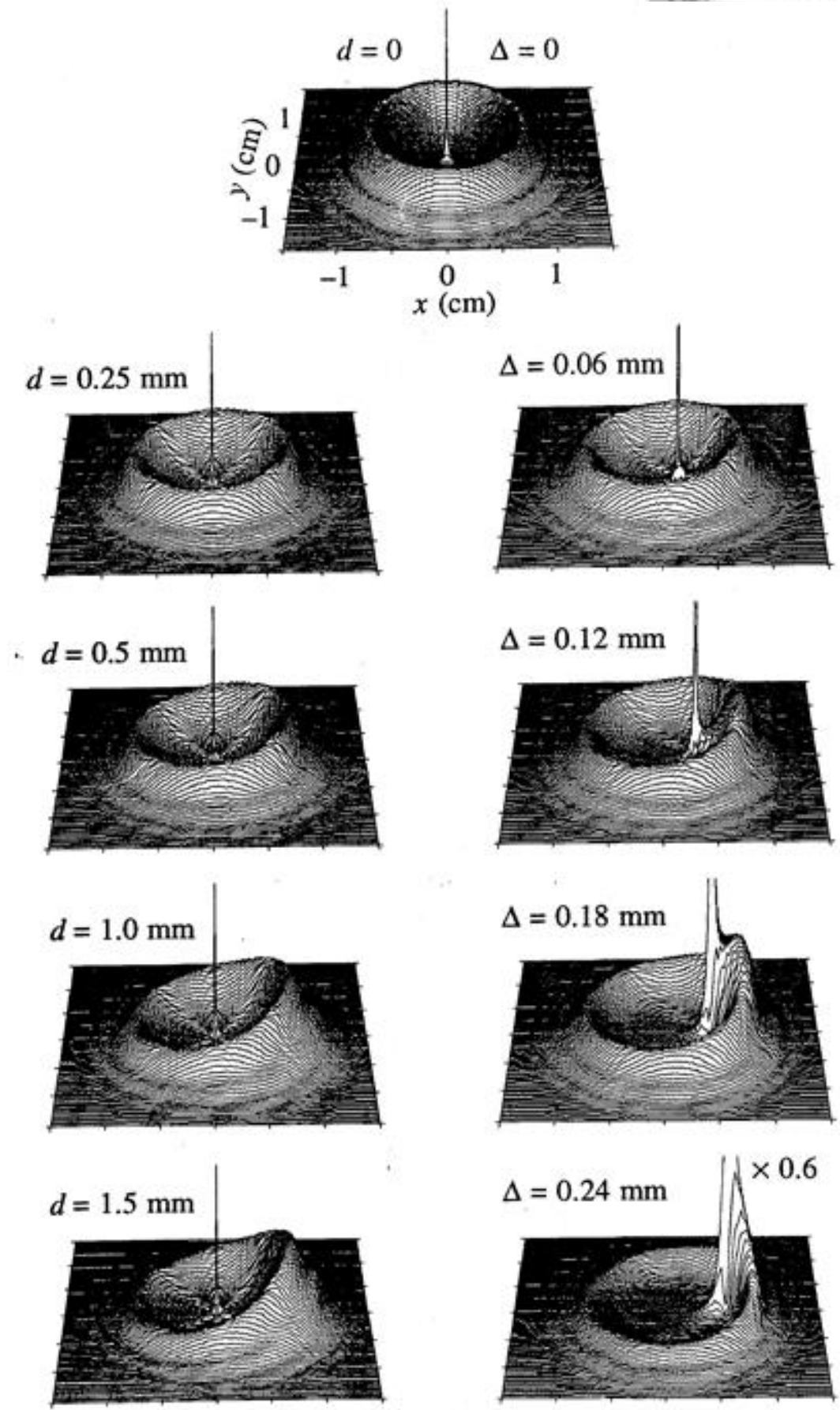

Figure 4. Effect of increasing displacement (left) and decenter (right) on two-dimensional intensity distribution in a particular plane $(\mathrm{z}=90 \mathrm{~cm})$. Calculation by Gauss-Legendre numerical integration.

While the increasing asymmetry in the intensity around the ring is quite similar in both cases, an interesting phenomenon, familiar to anyone who has worked with axicons, occurs with decenter; the peak moves towards one side of the ring while its intensity greatly increases. This latter effect is not visible in the figure, as the on the right-hand side of the figure had to be truncated. the maximum decenter illustrated $(0.24 \mathrm{~mm})$, the increase reaches almost an order of magnitude. 


\section{Experimental results}

A detailed experimental verification of the focusing characteristics of an axicon pair has been made. First, we tried to obtain a reference beams close as possible to a fundamental Gaussian which had the advantage (in comparison with a plane wave) of minimizing diffraction effects due to the outer edge of the second axicon. A well-know method for obtaining fundamentallooking beams consists in inserting an iris somewhere in the laser resonator. However, measurements of beam profiles from such lasers showed consistently important secondary lobes due to diffraction by the iris; these were eliminated by using a short near-confocal unapertured resonator (tube side notwithstanding) with a beam width much smaller than the size of the tube.

All measurements of the beam profiles were made by two complementary systems; a 64 element pyroelectrical detector array (Spiricon, model No. 200, with $170 \mu \mathrm{m}$ wide elements) and a travelling apertured $(100 \mu \mathrm{m})$ pyroelectrical detector (Molectron, model P1-53). Each had its own advantages in terms of speed, flexibility, and resolution. The detector array was used mainly for alignment purposes; all the final measurements were made with the travelling detector. Numerical data were recorded by a laboratory-made data acquisition system and stored for later treatment and analysis. All profiles were measured at least twice, and the measurements were repeated when any significant discrepancy was detected.

First, the profile of the reference beam was recorded in four planes to calculate its parameters and to verify that it was indeed fundamental. Figure 5 shows a comparison, in two planes, between the measured intensities (dots) and the best (in a least-square sense) Gaussian fit (solidline curve). Apart from a slight asymmetry (more visible in the plane $z=0$ ), the beam appeared to be fairly Gaussian. The content of higher order modes was evaluated also on the basis of a least-square method, based on the orthogonality of the Laguerre-Gauss higher order modes [30], which is suitable in the case of an incoherent superposition of modes. That analysis revealed no noticeable fraction of higher order modes.

Figure 6 shows the results of the measurements of the intensity distributions produced by the axicons, with an enlargement of the central zone of some distributions in the geometrical focal region. Notice that some amount of decenter and displacement of the beam is readily visible in the shapes of the distributions, in the form of asymmetries in the intensity of the sides of the ring and in the shape and location of the "central" peak. 


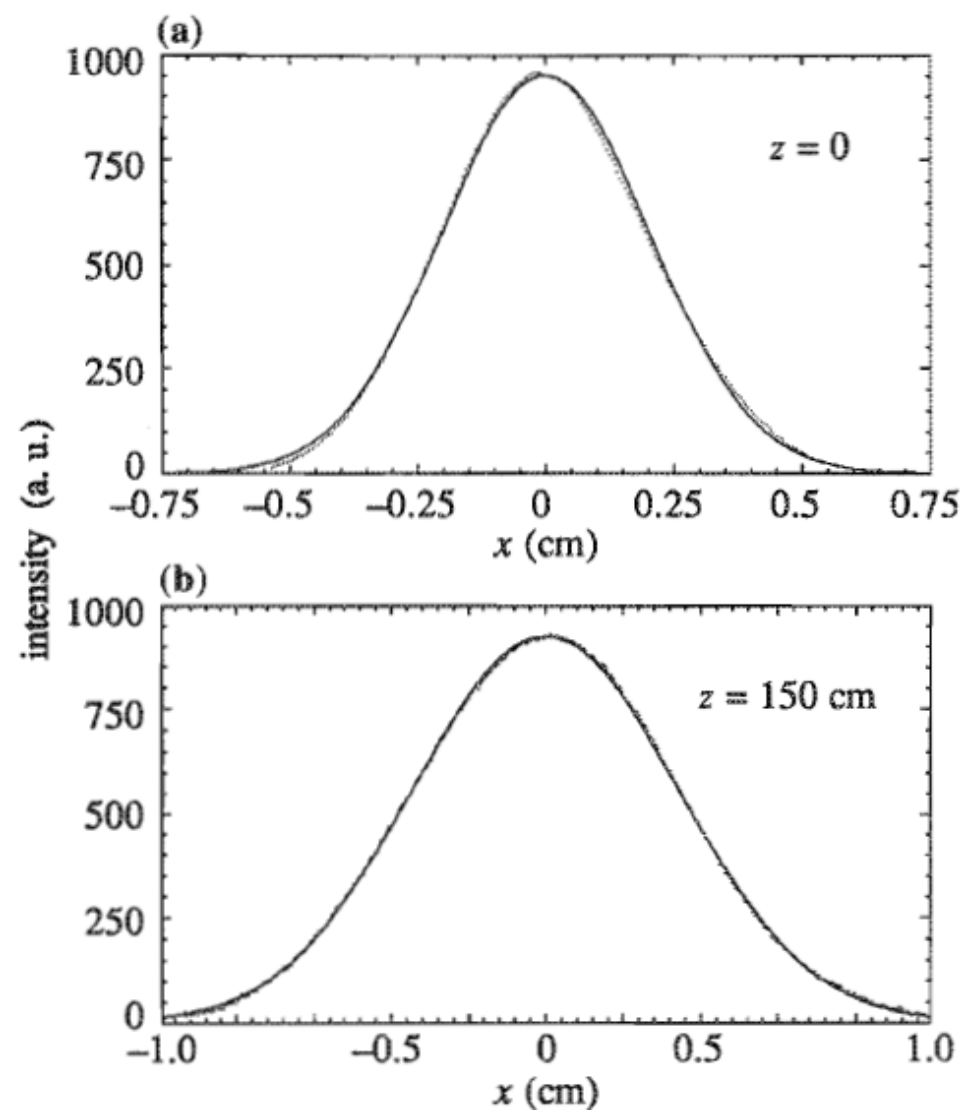

Figure 5. Measured profile of the reference beam in two planes (dots) superimposed on the best-fit Gaussian beam (solid line).

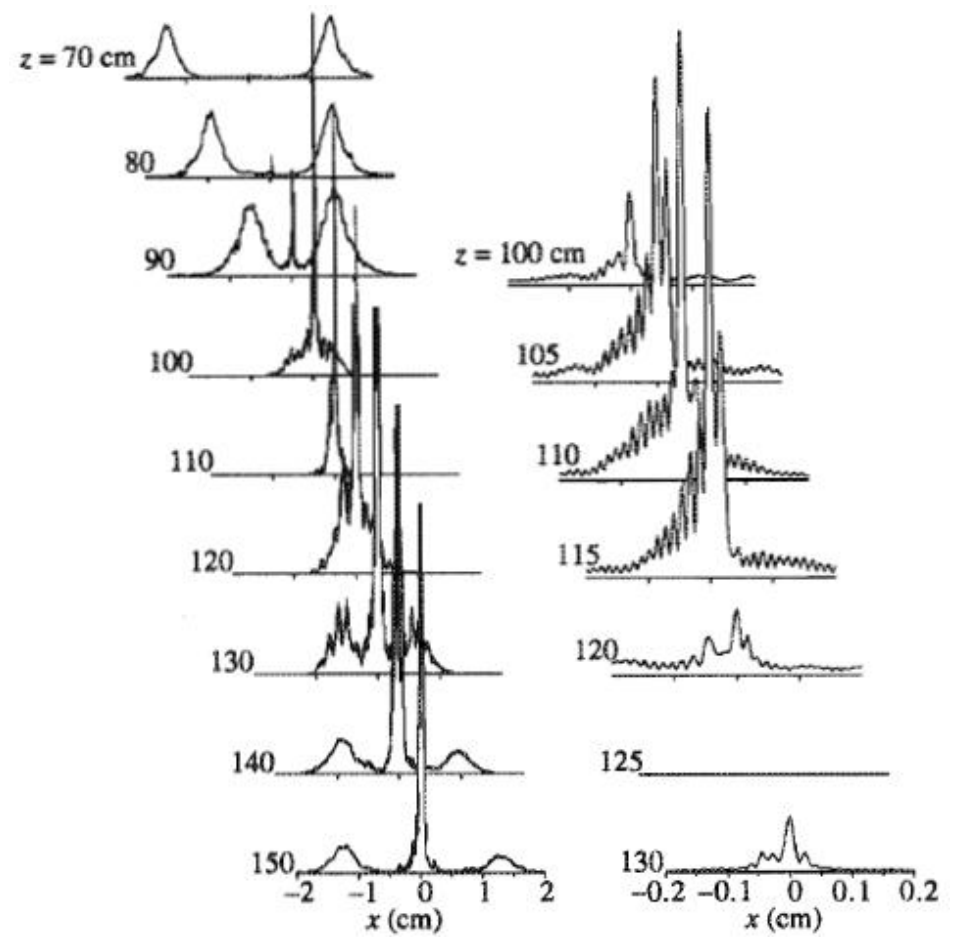

Figure 6. Global view of measured intensity distributions in the $x$ plane and enlargement of the central part of the distribution in the focal region. The distribution in the plane $z=125 \mathrm{~cm}$ was not measured. 
For a comparison with numerical results, one has to consider individual planes. Four of them, covering various parts of the focal line, are shown in Figure 7.
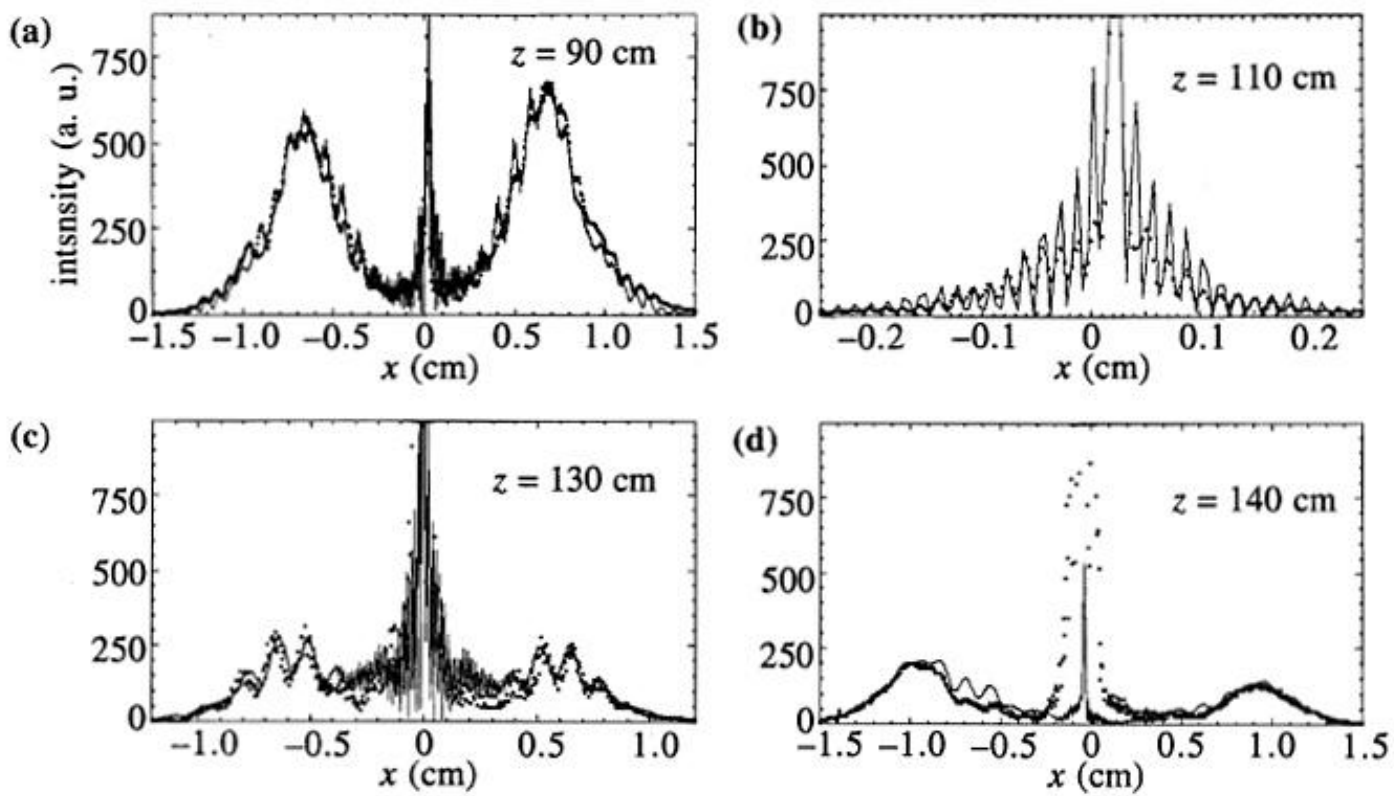

Figure 7. Comparison of measured and calculated distributions in four planes. Numerical calculation by the projection method, taking into account the presence of the supporting mounts of the first axicon.

By adjusting the decenter and the displacement of the beam, the main features of the distributions are well reproduced, as one can see. Notice that to obtain the large ripples in the sides of the ring (which are particularly visible at $z=90$ and $130 \mathrm{~cm}$ ), we had to include in the numerical calculations the presence of the cross-shaped supporting mount of the first axicon; this entailed the use of the projection method. Although it intercepted just a small fraction $(<2 \%)$ of the beam intensity, the mount, whose arms had been rotated by about $45^{\circ}$ to minimize obscuration along the measurement axis, clearly produced significant diffraction effects, as those ripples were almost completely absent in profiles obtained by previous calculations that had not taken its presence into account. The difference in the location of these structures can be attributed to the uncertainty in the mount's arms rotation angle; calculations reveal the these undulations shift by about half a period when this angle is changed by as little as $2^{\circ}$; these differences may also be the result of the slight asymmetry in the incoming beam intensity (which has to be associated with a corresponding asymmetry in the phase profile).

The main discrepancies concern the height (not always represented in the figures) and the width (especially in the plane $z=140 \mathrm{~cm}$ ) of the central peak, whose measured values are, respectively, much lower and much higher than the results of the numerical calculations. The first of these discrepancies disappears almost entirely if one applies to the data a convolution function accounting for the finite size of the detector aperture. Possible "figure" or "finish" defects of the axicons can also contribute, but to a lesser extent, to the same phenomenon . Crude estimates of the reduction of the peak intensity based on Kirchhoff-scattering theory [31] for finish errors, and numerical calculations for geometrical defects give $5 \%$ for the former (assuming on each surface a $\lambda / 40 \mathrm{rms}$ small-scale height variation with Gaussian distribution) and less than $1 \%$ for the latter (assuming a large-scale error of the order of $1 \mu \mathrm{m}$ ). 
The second discrepancy is more difficult to interpret. The intensity of the central part of the beam, after the focal plane, is due to the radiation in the "wings" of the incoming beam. A larger central peak thus suggests a beam with increased wings; this occurs with a multimode beam, a possibility we had ruled out on the grounds of the analysis of the measured beam shape discussed above. Indeed, calculations with various (incoherent) combinations of the first two modes produce distributions with large central peaks, but other features make them extremely different from the distributions of Figs. 7c and 7d.

Finally, a somewhat speculative hypothesis was put to the test. We assumed the presence of astigmatism in the incoming Gaussian beam. By increasing by a factor of 2.5 , in the exit plane of the axicons, the radius of curvature of the beam along the $y$ axis, we were able to greatly improve the fit between the experimental data and the calculations in the planes $z=130$ and $140 \mathrm{~cm}$ (Figure 8) while the other distributions, for which the width of the peak was already well reproduced, remained almost unchanged. Unfortunately, the experimental setup did not permit us to measure the beam profile along the two axes, and this hypothesis remains tentative. Such an astigmatism could be attributed to radial gain gradients in the laser medium, which are known to produce lensing effects [32], combined with the noncoincidence of the respective axes of the tube of the laser and its resonator. In fact, one had to realign a carefully aligned (by means of a He-Ne laser beam) resonator to obtain a Gaussian-shaped output; repeated attempts always resulted in a symmetric donut-shaped mode suggesting a lower gain near the axis.

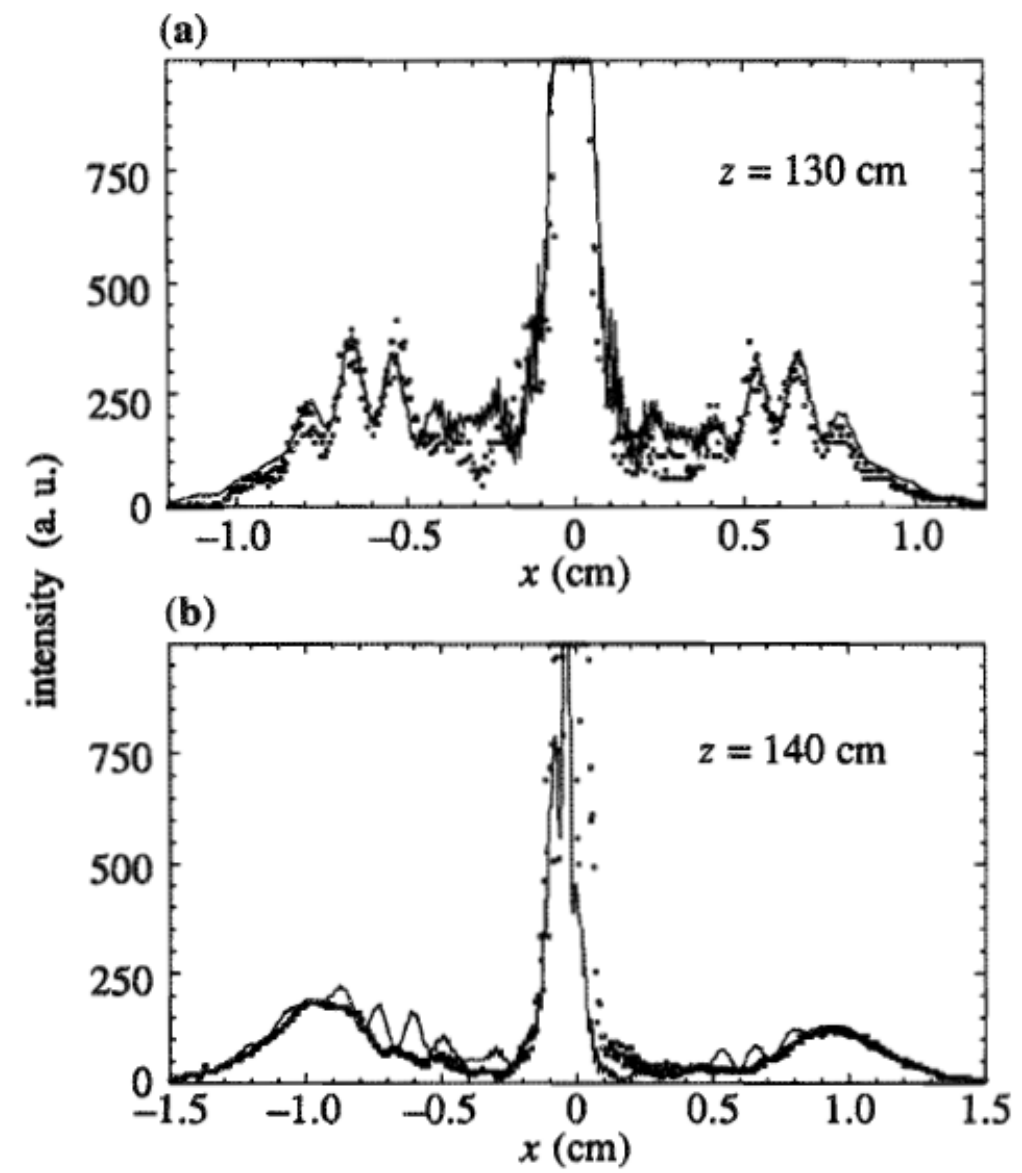

Figure 8. Comparison of measured and calculated distributions in the planes $z=130$ and $140 \mathrm{~cm}$. Same calculation as in Figs. 7c and 7d, but with astigmatic incoming beam (radius of curvature in the exit plane of the axicons increased by a factor 2.5 along the $y$ axis). 


\section{Conclusion}

We studied in detail, both theoretically (mainly numerically) and experimentally, the intensity distributions produced by focusing a Gaussian beam with a pair of reflective axicons (reflaxicon). Some important alignment errors (from an experimental point of view) were included in the numerical calculations and their effects on the shapes of the distributions were studied. On the experimental side, special care was taken to obtain a reference beam as close as possible to a fundamental Gaussian beam. The main characteristics as well as finer details of the measured profiles are well reproduced by the numerical calculations, if one accepts a few reasonable assumptions.

The calculation methods described here could thus be used with a fair degree of confidence to predict the intensity distributions in any experiment that uses axicons as focusing components. Especially, in experiments like direct or indirect optical pumping and long-plasma-line production, the application of one of these methods would be the starting point of the analysis of the spatial characteristics of the interaction.

\section{Acknowledgements}

This work has been supported in part by NSERC (The Natural Sciences and Engineering Research Council of Canada) and Fonds FCAR (Formation de chercheurs et aide a la recherche) of Québec.

\section{References}

1. J. H. McLeod. J. Opt. Soc. Am. 44, 592 (1954).

2. J. O. Stoner, Jr. Appl. Opt. 9, 53 (1970).

3. W. N. Peters and A. M. Ledger. Appl. Opt. 9, 1435 (1970).

4. O. Bryngdahl. J. Opt. Soc. Am. 61, 171 (1971).

5. Y. I. Balykin, V. S. Lethokov, Y. G. Minogin, Yu. Y. Rozhdestvensky, and A. I. Sidorov. J. Opt. Soc. Am. B2, 1776 (1985).

6. M. Florjanczyk and R. Tremblay. Opt. Commun. 73,448 (1989).

7. M.A. Saifi and M. J. Andrejco. Proc. Soc. Photo-Opt. Instrum. Eng. 668, 158 ( 1986).

8. W. Bobak, J. Jabczynski, Z. Jankiewicz, W. Nowakowski and J. Jasny. Opt. Appl. 17, 113 (1987).

9. F. P. Schafer. Appl Phys. B39, 1 (1986).

10. G. Kuhnle, G. Marowski and G. A. Reider. Appl. Opt. 27, 2666 (1988).

11. R. Tremblay, Y. D’Astous, G. Roy and M. Blanchard. Opt. Commun. 28, 193 (1979).

12. V. V. Korobkin, M. Yu. Marin, V. I. Pil'skii, L. Ya. Polonskii, and L. N. Pyatnitskii. Sov. J. Quantum. Electron. (Engl. Transl.), 15, 631 (1985). 
13. V. V. Korobkin, L. Ya. Polonskii, V. P. Poponin, and L. N. Pyatnitskii. Sov. J. Quantum. Electron. (Engl. Transl.), 16, 178 (1986).

14. O. G. Ivanov, R. I. Okunev, L. N. Pakhomov, V. Yu. Petrun'kin, L. Ya. Polonskii, and L. N. Pyatnitskii. Sov. Phys. Tech. Phys. (Engl. Transl.), 32, 1212 (1987).

15. W. R. Edmonds. Appl. Opt. 12, 1940 (1973).

16. M. Rioux, R. Tremblay, and P. A. Bélanger. Appl. Opt. 17, 1532 (1978).

17. A. E. Siegman. Lasers. University science books, Mill Valley, CA. 1986.

18. A. Chandonnet, R. Bazin, C. Sirois, and P. A. Bélanger. Lasers in medicine and surgery, 12, 264 (1992).

19. S. Fujiwara. J. Opt. Soc. Am. 52, 287 (1962).

20. J. W. Y. Lit and E. Brannen. J. Opt. Soc. Am. 60, 370 (1970).

21. J. W. Y. Lit. J. Opt. Soc. Am. 60, 1001 (1970).

22. J. W. Y. Lit and R. Tremblay. J. Opt. Soc. Am. 63, 445 (1973).

23. G. Roy and R. Tremblay. Opt. Commun. 34, 1 (1980).

24. M. Y. Perez, C. Gomez-Reino, and J. M. Cuadrado. Opt. Acta 33, 1161 (1986).

25. W. Swantner. Opt. Eng. 21, 333 (1982).

26. P. A. Bélanger and M. Rioux. Appl. Opt. 17, 1080 (1978).

27. M. Born and E. Wolf. Principles of optics. 6th ed. Pergamon Press, Oxford. 1980.

28. S. Solimeno, B. Crosignani, and P. Di Porto. Guiding, diffraction and confinement of optical radiation. Academic Press, Orlando. 1986.

29. A. V. Oppenheim, G. V. Frisk, and D. R. Martinez. J. Acoust. Soc. Am. 68, 523 (1980).

30. M. Couture. Ph. D. Thesis, Université Laval, Sainte Foy, Qué. 1991.

31. J. A. Ogilvy. Theory of wave scattering from random surfaces. Institute of Physics Publishing, Philadelphia. 1991. p. 88.

32. G. Stephan and M. Trumper. Phys.Rev. A: Gen. Phys. 28, 2344 (1983). 